\title{
Remarks on uniform attractors for the 3D non- autonomous Navier-Stokes-Voight equations
}

Yiwen Dou ${ }^{1,2}$, Xinguang Yang ${ }^{3^{*}}$ and Yuming Qin ${ }^{4}$

\author{
* Correspondence: \\ yangxinguangyxg@yahoo.cn \\ ${ }^{3}$ College of Mathematics and \\ Information Science, Henan Normal \\ University, Xinxiang, 453007, \\ People's Republic of China \\ Full list of author information is \\ available at the end of the article
}

\begin{abstract}
In this paper, we show the existence of pullback attractors for the non-autonomous Navier-Stokes-Voight equations by using contractive functions, which is more simple than the weak continuous method to establish the uniformly asymptotical compactness in $H^{1}$ and $H^{2}$.

2010 Mathematics Subject Classification: 35D05; 35M10
\end{abstract}

Keywords: Navier-Stokes-Voight equations, processes, contractive functions, uniform attractors

\section{Introduction}

Let $\Omega \subset R^{3}$ be a bounded domain with sufficiently smooth boundary $\partial \Omega$. We consider the non-autonomous 3D Navier-Stokes-Voight (NSV) equations that govern the motion of a Klein-Voight linear viscoelastic incompressible fluid:

$$
\begin{aligned}
& u_{t}-v \Delta u-\alpha^{2} \Delta u_{t}+(u \cdot \nabla) u+\nabla p=f(t, x), \quad(x, t) \in \Omega \times R_{\tau}, \\
& \nabla \cdot u=0, \quad x \in \Omega, \quad t \in R_{\tau}, \\
& \left.u(t, x)\right|_{\partial \Omega}=0, \quad t \in R_{\tau}, \\
& u(\tau, x)=u_{\tau}(x), \quad x \in \Omega, \quad \tau \in R_{\tau} .
\end{aligned}
$$

Here $u=u(t, x)=\left(u_{1}(t, x), u_{2}(t, x), u_{3}(t, x)\right)$ is the velocity vector field, $p$ is the pressure, $v>0$ is the kinematic viscosity, and the length scale $\alpha$ is a characterizing parameter of the elasticity of the fluid.

When $\alpha=0$, the above system reduce to the well-known 3D incompressible NavierStokes system:

$$
\begin{aligned}
& u_{t}-v \Delta u+(u \cdot \nabla) u+\nabla p=f(t, x), x \in \Omega, t \in R_{\tau}, \\
& \nabla \cdot u=0, x \in \Omega, t \in R_{\tau} .
\end{aligned}
$$

For the well-posedness of 3D incompressible Navier-Stokes equations, in 1934, Leray [1-3] derived the existence of weak solution by weak convergence method; Hopf [4] improved Leray's result and obtained the familiar Leray-Hopf weak solution in 1951. Since the 3D Navier-Stokes equations lack appropriate priori estimate and the strong

\section{Springer}

(C) 2011 Dou et al; licensee Springer. This is an Open Access article distributed under the terms of the Creative Commons Attribution License (http://creativecommons.org/licenses/by/2.0), which permits unrestricted use, distribution, and reproduction in any medium, provided the original work is properly cited. 
nonlinear property, the existence of strong solution remains open. For the infinitedimensional dynamical systems, Sell [5] constructed the semiflow generated by the weak solution which lacks the global regularity and obtained the existence of global attractor of the 3D incompressible Navier-Stokes equations on any bounded smooth domain. Chepyzhov and Vishik [6] investigated the trajectory attractors for 3D nonautonomous incompressible Navier-Stokes system which is based on the works of Leray and Hopf. Using the weak convergence topology of the space $H$ (see below for the definition), Kapustyan and Valero [7] proved the existence of a weak attractor in both autonomous and non-autonomous cases and gave a existence result of strong attractors. Kapustyan, Kasyanov and Valero [8] considered a revised 3D incompressible Navier-Stokes equations generated by an optimal control problem and proved the existence of pullback attractors by constructing a dynamical multivalued process.

However, the infinite-dimensional systems for 3D incompressible Navier-Stokes equations have not yet completely resolved, so many mathematicians pay attention to this challenging problem. In this regard, Kalantarov and Titi [9] investigated the Navier-Stokes-Voight equations as an inviscid regularization of the 3D incompressible Navier-Stokes equations, and further obtained the existence of global attractors for Navier-Stokes-Voight equations. Recently, Qin, Yang and Liu [10] showed the existence of uniform attractors by uniform condition-(C) and weak continuous method to obtain uniformly asymptotical compactness in $H^{1}$ and $H^{2}$, Yue and Zhong [11] investigated the attractors for autonomous and nonautonomous 3D Navier-Stokes-Voight equations in different methods. More details about the infinite-dimensional dynamics systems, we can refer to [12-27].

Using the contractive functions, we have in this paper established the uniformly asymptotical compactness of the processes $\{U(t, \tau)\}(t \geq \tau, \tau \in R)$ to obtain the existence of the uniform attractor of the 3D non-autonomous NSV equations.

Main difficulties we encountered are as follows:

(1) how to obtain a contractive function,

(2) how to deduce the uniformly asymptotical compactness from a contractive function,

(3) how to obtain the convergence of contractive function.

\section{Main results}

Notations: Throughout this paper, we set $R_{\tau}=[\tau,+\infty), \tau \in R$. C stands for a generic positive constant, depending on $\Omega$, but independent of $t . L^{p}(\Omega)(1 \leq p \leq+\infty)$ is the generic Lebesgue space, $H^{s}(\Omega)$ is the general Sobolev space. We set $E:=\left\{u \mid u \in\left(C_{0}^{\infty}(\Omega)\right)^{3}, \operatorname{divu}=0\right\}, H, V, W$ is the closure of the set $E$ in the topology of $\left(L^{2}(\Omega)\right)^{3},\left(H^{1}(\Omega)\right)^{3},\left(H^{2}(\Omega)\right)^{3}$ respectively. " $\triangle$ " stands for weak convergence of sequence.

Let $\Sigma \subseteq L_{\text {loc }}^{2}\left(R, L^{2}(\Omega)\right)$ be the hull of $f_{0}$ as a symbol space:

$$
\Sigma=H_{+}\left(f_{0}\right)=\left[f_{0}(t+h) \mid h \in R\right]_{L_{\text {loc }}^{2}\left(R, L^{2}(\Omega)\right)}
$$

for all $f_{0} \in L_{l o c}^{2}\left(R, L^{2}(\Omega)\right)$, where $[\cdot]_{L_{l o c}^{2}\left(R, L^{2}(\Omega)\right)}$ denotes the closure in the topology of $L_{l o c}^{2}\left(R, L^{2}(\Omega)\right)$.

Under the assumptions of the initial data, the problem (1.1)-(1.4) has a global solution $u \in C([\tau,+\infty), V) . U_{f}\left(t, \tau, u_{\tau}\right): V \rightarrow V$ denotes the processes generated by the 
global solutions and satisfies

$$
\begin{aligned}
& u\left(t, \tau ; u_{\tau}\right)=U_{f}\left(t, \tau, u_{\tau}\right) u_{\tau} \\
& U_{f}\left(t, s, u_{\tau}\right) \cdot U_{f}\left(s, \tau, u_{\tau}\right)=U_{f}\left(t, \tau, u_{\tau}\right), \forall u_{\tau} \in \Sigma, t \geq \tau, \tau \geq 0, \\
& U_{f}(\tau, \tau)=I d, \forall \tau \in R .
\end{aligned}
$$

Let $\{T(s)\}$ be the translation semigroup on $\Sigma$, we see that the family of processes $\left\{U_{f}\right.$ $(t, \tau)\}(f \in \Sigma)$ satisfies the translation identity if

$$
\begin{aligned}
& U_{f}(t+s, \tau+s)=U_{T(s) f}(t, \tau), \forall f \in \Sigma, t \geq \tau, \tau \in R, \\
& T(s) \Sigma=\Sigma, \forall s \geq 0 .
\end{aligned}
$$

Next, we recall a simple method to derive uniformly asymptotical compactness which can be found in [28].

Definition 2.1 Let $X$ be a Banach space and $B$ be a bounded subset of $X, \Sigma$ be a symbol space. We call a function $\varphi(\cdot, \cdots, \cdot)$ defined on $(X \times X) \times(\Sigma \times \Sigma)$ to be a contractive function on $B \times B$ if for any sequence $\left\{x_{n}\right\}_{n=1}^{\infty} \subset B$ and any $\left\{g_{n}\right\} \subset \Sigma$, there are subsequences $\left\{x_{n_{k}}\right\}_{k=1}^{\infty} \subset\left\{x_{n}\right\}_{n=1}^{\infty}$ and $\left\{g_{n_{k}}\right\}_{k=1}^{\infty} \subset\left\{g_{n}\right\}_{n=1}^{\infty}$ such that

$$
\lim _{k \rightarrow \infty} \lim _{l \rightarrow \infty} \phi\left(x_{n_{k}}, x_{n_{l}} ; g_{n_{k}}, g_{n_{l}}\right)=0 .
$$

We denote the set of all contractive functions on $B \times B$ by $\operatorname{Contr}(B, \Sigma)$.

Lemma 2.2 Let $\left\{U_{f}(t, \tau)\right\}(f \in \Sigma)$ be a family of processes satisfying the translation identity (2.5) and (2.6) on Banach space $X$ and has a bounded uniform (w.r.t $f \in \Sigma$ ) absorbing set $B_{0} \subset X$. Moreover, assume that for any $\varepsilon>0$, there exist $T=T\left(B_{0}, \varepsilon\right)$ and $\varphi_{T} \in \operatorname{Contr}\left(B_{0}, \Sigma\right)$ such that

$$
\begin{aligned}
\left\|U_{f_{1}}(T, 0) x-U_{f_{2}}(T, 0) y\right\| \leq \varepsilon+\phi_{T}\left(x, y ; f_{1}, f_{2}\right), & \\
& \forall x, y \in B_{0}, \forall f_{1}, f_{2} \in \Sigma .
\end{aligned}
$$

Then $\left\{U_{f}(t, \tau)\right\}(f \in \Sigma)$ is uniformly (w.r.t. $\left.f \in \Sigma\right)$ asymptotically compact in $X$.

Theorem 2.3 Assume that $f \in \Sigma \subseteq L^{2}(R, H), u_{\tau} \in V$, then the problem (1.1)-(1.4) possesses uniform attractors $\mathcal{A}_{f}^{1}(t)$ in $V$.

Theorem 2.4 Assume that $f \in \Sigma \subseteq L^{2}(R, H), u_{\tau} \in W$, then the problem (1.1)-(1.4) possesses uniform attractors $\mathcal{A}_{f}^{2}(t)$ in $W$.

\section{Proof of Theorem 2.3}

In this section, we shall prove Theorem 2.3 by two steps as follows, the first one is to get the existence of an absorbing ball, the second is to prove the asymptotical compactness by means of a contractive function.

From the property of solutions, we can easily derive that the set class $\left\{U_{f}\left(t, \tau, u_{\tau}\right)\right\}(\tau$, $\leq t)$ is a process in $V$ for all $\tau \leq t$. Moreover, the mapping $U_{f}\left(t, \tau, u_{\tau}\right): V \rightarrow V$ is continuous.

Lemma 3.1 We assume that $\left\{u_{\tau}^{n}\right\} \subset V, u_{\tau} \in V$ and $u_{\tau}^{n} \rightarrow u_{\tau}, f^{n} \rightarrow f$ in $L^{2}(R, H)$, then 


$$
\begin{aligned}
\begin{aligned}
U_{f^{n}}\left(t, \tau, u_{\tau}^{n}\right) u_{\tau}^{n} \rightarrow & U_{f}\left(t, \tau, u_{\tau}\right) u_{\tau} \\
& \text { weakly star in } L^{\infty}((\tau, T) ; H), \forall t \geq \tau, \\
U_{f^{n}}\left(\cdot, \tau, u_{\tau}^{n}\right) u_{\tau}^{n} \rightarrow & U_{f}\left(\cdot, \tau, u_{\tau}\right) u_{\tau} \\
& \text { weakly in } L^{2}(\tau, T ; V), \forall t \geq \tau .
\end{aligned}
\end{aligned}
$$

Proof. From the boundedness of the solutions in corresponding topological spaces, we easily conclude the results.

Lemma 3.2 Assume $f \in L^{2}(R, H), u_{\tau} \in V$, then there exists a uniform (w.r.t. $f \in \Sigma$ ) absorbing set $B_{0}$ of processes $\left\{U_{f}\left(t, \tau, u_{\tau}\right)\right\}$.

Proof. For all $u \in V$, multiplying both sides of (1.1) with $u$ and noting that $((u \cdot \nabla) u$, $u)=0$, we derive

$$
\begin{gathered}
\frac{\mathrm{d}}{\mathrm{d} t}\left(\|u(t)\|^{2}+\alpha^{2}\|\nabla u(t)\|^{2}\right)+2 v\|\nabla u(t)\|^{2} \leq 2(f(t), u(t)) \\
\leq 2 v\|\nabla u(t)\|^{2}+\frac{2}{v \lambda}\|f(t)\|^{2} .
\end{gathered}
$$

Consequently, for all $\tau \in R$, there holds

$$
\|u(t)\|^{2}+\alpha^{2}\|\nabla u(t)\|^{2} \leq\left(\left\|u_{\tau}\right\|^{2}+\alpha^{2}\|\nabla u(\tau)\|^{2}\right)+\frac{2}{v \lambda} \int_{\tau}^{t}\|f(\xi)\|^{2} \mathrm{~d} \xi .
$$

Consider the property of the functional $\langle\cdot \cdot \cdot\rangle+\alpha^{1}\langle\nabla \cdot, \nabla \cdot\rangle$, we get

$$
C_{1}\|\cdot\|_{V}^{2} \leq\langle\cdot, \cdot\rangle+\alpha^{2}\langle\nabla \cdot, \nabla \cdot\rangle \leq C_{2}\|\cdot\|_{V}^{2}, 0<C_{1} \leq C_{2},
$$

and there exists a constant $C_{0}$ satisfying $C_{1} \leq C_{0} \leq C_{2}$, such that

$$
C_{0}\|\cdot\|_{V}^{2}=\langle\cdot, \cdot\rangle+\alpha^{2}\langle\nabla \cdot, \nabla \cdot\rangle .
$$

Setting the radius $r^{2}=\left\|u_{\tau}\right\|^{2}+\alpha^{2}\left\|\nabla u_{\tau}\right\|^{2}$, we easily get that there exists a constant $C>0$ such that

$$
\left\|U_{f}\left(t, \tau, u_{\tau}\right)\right\|_{V}^{2} \leq C r^{2}+\frac{2 C}{v \lambda} \int_{-\infty}^{t}\|f(\xi)\|^{2} \mathrm{~d} \xi,
$$

for all $u_{\tau} \in V, t \geq \tau$.

Setting

$$
r^{2} \leq \frac{2}{v \lambda} \int_{-\infty}^{t}\|f(\xi)\|^{2} \mathrm{~d} \xi
$$

then we denote $R$ the nonnegative number given by

$$
R^{2}=\frac{2 C}{v \lambda} \int_{-\infty}^{t}\|f(\xi)\|^{2} \mathrm{~d} \xi
$$

and consider the family of closed balls $B_{0}$ in $V$ defined by

$$
B_{0}=\left\{v \in V \mid\|v\|_{V} \leq 2 R\right\} .
$$

It is straightforward to check that $B_{0}$ is a uniform absorbing ball for the processes $\left\{U_{f}\left(t, \tau, u_{\tau}\right)\right\}$. 
Lemma 3.3 Under the condition of $f \in L^{2}(R, H)$, the process $\left\{U_{f}\left(t, \tau, u_{\tau}\right)\right\}$ generated by the global solutions for problem (1.1)-(1.4) is uniformly asymptotically compact in $V$.

Proof. For any initial data $u_{\tau}^{i} \in B_{0}(i=1,2)$, let $u^{i}(t, x)$ be the corresponding solutions to the symbols $f^{i}$ with $u_{\tau}^{i}$, that is, $u^{i}(t)$ is the solution of the problem:

$$
\begin{aligned}
& u_{t}-\alpha^{2} \Delta u_{t}-v \Delta u+(u \cdot \nabla) u+\nabla p=f^{i}(t, x), \quad x \in \Omega, \quad t \in R_{\tau} \\
& \operatorname{divu}=0, \quad t \in R_{\tau} \\
& \left.u(t, x)\right|_{\partial \Omega}=0, \quad t \in R_{\tau} \\
& u(\tau, x)=u_{\tau}^{i}(x), \quad \tau \in R .
\end{aligned}
$$

Denote

$$
w(t)=u^{1}(t)-u^{2}(t)
$$

then $w(t)$ satisfies the equivalent abstract equations

$$
\begin{aligned}
& w_{t}+\alpha A w_{t}-v A w+B\left(u^{1}\right)-B\left(u^{2}\right)=f^{1}(t, x)-f^{2}(t, x), \\
& \operatorname{div} w=0, \\
& \left.w(t, x)\right|_{\partial \Omega}=0, \\
& w(\tau, x)=u_{\tau}^{1}(x)-u_{\tau}^{2}(x), \quad \tau \in R,
\end{aligned}
$$

where $B(u)=(u \cdot \nabla) u, p$ has disappeared by the projection operator $P$.

Setting

$$
E_{w}(t)=\frac{1}{2} \int_{\Omega}|w(t)|^{2} \mathrm{~d} x+\frac{\alpha^{2}}{2} \int_{\Omega}|\nabla w(t)|^{2} \mathrm{~d} x .
$$

Multiplying (3.13) by $w$ and integrating over $[s, T] \times \Omega$, we deduce

$$
\begin{aligned}
& E_{w}(T)-E_{w}(s)+v \int_{s}^{T} \int_{\Omega}|\nabla w(h)|^{2} \mathrm{~d} x \mathrm{~d} h \\
& +\int_{s}^{T} \int_{\Omega}\left(B\left(u^{1}(h)\right)-B\left(u^{2}(h)\right)\right) w(h) \mathrm{d} x \mathrm{~d} h \\
& =\int_{s}^{T} \int_{\Omega}\left(f^{1}(h)-f^{2}(h)\right) w(h) \mathrm{d} x \mathrm{~d} h,
\end{aligned}
$$

where $\tau \leq s \leq T$. Then we have

$$
\begin{gathered}
v \int_{\tau}^{T} \int_{\Omega}|\nabla w(h)| \mathrm{d} x \mathrm{~d} h \leq E_{w}(\tau)-\int_{\tau}^{T} \int_{\Omega}\left(B\left(u^{1}(h)\right)-B\left(u^{2}(h)\right)\right) w(h) \mathrm{d} x \mathrm{~d} h \\
+\int_{\tau}^{T} \int_{\Omega}\left(f^{1}(h)-f^{2}(h)\right) w(h) \mathrm{d} x \mathrm{~d} h .
\end{gathered}
$$


Hence,

$$
\begin{aligned}
\int_{\tau}^{T} E_{w}(s) \mathrm{d} s= & \int_{\tau}^{T}\left(\frac{1}{2} \int_{\Omega}-w(s)-{ }^{2} \mathrm{~d} x+\frac{\alpha^{2}}{2} \int_{\Omega}-\nabla w(s)-{ }^{2} \mathrm{~d} x\right) \mathrm{d} s \\
\leq & C \int_{\tau}^{T} \int_{\Omega}-\nabla w(s)-{ }^{2} \mathrm{~d} x \mathrm{~d} s \\
\leq & C\left[E_{w}(\tau)-\int_{\tau}^{T} \int_{\Omega}\left(B\left(u^{1}(s)\right)-B\left(u^{2}(s)\right)\right) w(s) \mathrm{d} x \mathrm{~d} s\right. \\
& \left.+\int_{\tau}^{T} \int_{\Omega}\left(f^{1}(s)-f^{2}(s)\right) w(s) \mathrm{d} x \mathrm{~d} s\right] .
\end{aligned}
$$

Integrating (3.18) over $[\tau, T]$ with respect to $s$, we get

$$
\begin{aligned}
T E_{w}(T) & +v \int_{\tau}^{T} \int_{s}^{T} \int_{\Omega}|\nabla w(h)| \mathrm{d} x \mathrm{~d} h \mathrm{~d} s \\
\leq & \int_{\tau}^{T} E_{w}(s) \mathrm{d} s-\int_{\tau}^{T} \int_{s}^{T} \int_{\Omega}\left(B\left(u^{1}(h)\right)-B\left(u^{2}(h)\right)\right) w(h) \mathrm{d} x \mathrm{~d} h \mathrm{~d} s \\
& +\int_{\tau}^{T} \int_{s}^{T} \int_{\Omega}\left(f^{1}(h)-f^{2}(h)\right) w(h) \mathrm{d} x \mathrm{~d} h \mathrm{~d} s \\
\leq & C\left[E_{w}(\tau)-\int_{\tau}^{T} \int_{\Omega}\left(B\left(u^{1}(s)\right)-B\left(u^{2}(s)\right)\right) w(s) \mathrm{d} x \mathrm{~d} s\right. \\
& \left.+\int_{\tau}^{T} \int_{\Omega}\left(f^{1}(s)-f^{2}(s)\right) w(s) \mathrm{d} x \mathrm{~d} s\right] \\
& -\int_{\tau}^{T} \int_{s}^{T} \int_{\Omega}\left(B\left(u^{1}(h)\right)-B\left(u^{2}(h)\right)\right) w(h) \mathrm{d} x \mathrm{~d} h \mathrm{~d} s \\
& +\int_{\tau}^{T} \int_{s}^{T} \int_{\Omega}\left(f^{1}(h)-f^{2}(h)\right) w(h) \mathrm{d} x \mathrm{~d} h \mathrm{~d} s .
\end{aligned}
$$

If we set

$$
\begin{aligned}
& C_{0}=C E_{w}(\tau), \\
& \begin{aligned}
\phi\left(u_{0}^{1}, u_{0}^{2} ; g^{1}(t), g^{2}(t)\right)=C[- & \int_{\tau}^{T} \int_{\Omega}\left(B\left(u^{1}(s)\right)-B\left(u^{2}(s)\right)\right) w(s) \mathrm{d} x \mathrm{~d} s \\
& \left.+\int_{\tau}^{T} \int_{\Omega}\left(f^{1}(s)-f^{2}(s)\right) w(s) \mathrm{d} x \mathrm{~d} s\right] \\
& -\int_{\tau}^{T} \int_{s}^{T} \int_{\Omega}\left(B\left(u^{1}(h)\right)-B\left(u^{2}(h)\right)\right) w(h) \mathrm{d} x \mathrm{~d} h \mathrm{~d} s \\
& +\int_{\tau}^{T} \int_{s}^{T} \int_{\Omega}\left(f^{1}(h)-f^{2}(h)\right) w(h) \mathrm{d} x \mathrm{~d} h \mathrm{~d} s,
\end{aligned}
\end{aligned}
$$

then we have

$$
E_{w}(T) \leq \frac{C_{0}}{T}+\frac{1}{T} \phi\left(u_{0}^{1}, u_{0}^{2} ; f^{1}(t), f^{2}(t)\right) .
$$

Since the family of processes has a uniformly bounded absorbing set, we choose $T$ large enough such that

$$
\frac{C_{0}}{T} \leq \varepsilon,
$$

i.e., $T \geq \frac{C_{0}}{\varepsilon}$. 
Let $u^{n}, u^{m}$ be the solutions with respect to the initial data $u_{0}^{n}, u_{0}^{m}$ and symbols $f^{n}(t)$, $f^{m}(t) \in \Sigma, m, n=1,2, \ldots$ respectively. Then from Lemma 3.1, we can derive

$$
\begin{aligned}
& \lim _{n \rightarrow \infty} \lim _{m \rightarrow \infty} \int_{\tau}^{T} \int_{s}^{T} \int_{\Omega}\left(f^{n}(h)-f^{m}(h)\right)\left(u^{n}(s)-u^{m}(s)\right) \mathrm{d} x \mathrm{~d} h \mathrm{~d} s=0, \\
& \lim _{n \rightarrow \infty} \lim _{m \rightarrow \infty} \int_{\tau}^{T} \int_{\Omega}\left(f^{n}(s)-f^{n}(s)\right)\left(u^{n}(s)-u^{m}(s)\right) \mathrm{d} x \mathrm{~d} s=0,
\end{aligned}
$$

and

$$
\begin{aligned}
& \lim _{n \rightarrow \infty} \lim _{m \rightarrow \infty} \int_{\tau}^{T} \int_{\Omega}\left(B\left(u^{n}(s)\right)-B\left(u^{m}(s)\right)\right) \\
& \times\left(u^{n}(s)-u^{m}(s)\right) \mathrm{d} x \mathrm{~d} s \\
& =\lim _{n \rightarrow \infty} \lim _{m \rightarrow \infty} \int_{s}^{T} \int_{\Omega}\left(\left(u^{n}(s) \cdot \nabla\right) u^{n}(s)-\left(u^{m}(s) \cdot \nabla\right) u^{m}(s)\right) \\
& \times\left(u^{n}(s)-u^{m}(s)\right) \mathrm{d} x \mathrm{~d} s \\
& =\lim _{n \rightarrow \infty} \lim _{m \rightarrow \infty} \int_{s}^{T} \int_{\Omega}\left(\left(\left(u^{n}(s)-u^{m}(s)\right) \cdot \nabla\right) u^{n}(s)-\left(u^{m}(s) \cdot \nabla\right)\right. \\
& \left.\times\left(u^{m}(s)-u^{n}(s)\right)\right) \times\left(u^{n}(s)-u^{m}(s)\right) \mathrm{d} x \mathrm{~d} s \\
& =0, \\
& \lim _{n \rightarrow \infty} \lim _{m \rightarrow \infty} \int_{\tau}^{T} \int_{s}^{T} \int_{\Omega}\left(B\left(u^{n}(h)\right)-B\left(u^{m}(h)\right)\right)\left(u^{n}(s)-u^{m}(s)\right) \mathrm{d} x \mathrm{~d} h \mathrm{~d} s \\
& =\lim _{n \rightarrow \infty} \lim _{m \rightarrow \infty} \int_{\tau}^{T} \int_{s}^{T} \int_{\Omega}\left(\left(u^{n}(h) \cdot \nabla\right) u^{n}(h)-\left(u^{m}(h) \cdot \nabla\right) u^{m}(h)\right) \\
& \times\left(u^{n}(s)-u^{m}(s)\right) \mathrm{d} x \mathrm{~d} h \mathrm{~d} s \\
& =\lim _{n \rightarrow \infty} \lim _{m \rightarrow \infty} \int_{\tau}^{T} \int_{s}^{T} \int_{\Omega}\left(\left(\left(u^{n}(h)-u^{m}(h)\right) \cdot \nabla\right) u^{n}(h)-\left(u^{m}(h) \cdot \nabla\right)\right. \\
& \left.\times\left(u^{m}(h)-u^{n}(h)\right)\right) \times\left(u^{n}(s)-u^{m}(s)\right) \mathrm{d} x \mathrm{~d} h \mathrm{~d} s \\
& =0 .
\end{aligned}
$$

Hence $\phi\left(u_{0}^{1}, u_{0}^{2} ; f^{1}(t), f^{2}(t)\right) \in \operatorname{Contr}\left(B_{0}, \Sigma\right)$ for the above $T$. By Lemma 2.2 and the property of the functional $\langle\cdot, \cdot\rangle+\alpha^{2}\langle\nabla \cdot, \nabla \cdot\rangle$, the conclusion holds.

Proof of Theorem 2.3 From Lemmas 3.1-3.3, we can deduce the result easily.

\section{Proof of Theorem 2.4}

Similarly to the proof of Theorem 2.3, we easily obtain that the set class $\left\{U_{f}\left(t, \tau, u_{\tau}\right)\right\}(\tau$ $\leq t)$ is a process in $W$ for all $\tau \leq t$. Moreover, the mapping $U_{f}\left(t, \tau, u_{\tau}\right): W \rightarrow W$ is continuous. If we assume that $\left\{u_{\tau}^{n}\right\}$ is a sequence in $W$ and weakly converges to $u_{\tau} \in W$, $f^{n} \rightarrow f$ in $L^{2}(R, H)$, then

$$
\begin{aligned}
& U_{f^{n}}\left(t, \tau, u_{\tau}^{n}\right) u_{\tau}^{n} \rightarrow U_{f}\left(t, \tau, u_{\tau}\right) u_{\tau} \text { weakly in } W, \forall \text { fixed } t \geq \tau, \\
& U_{f^{n}}\left(\cdot, \tau, u_{\tau}^{n}\right) u_{\tau}^{n} \rightarrow U_{f}\left(\cdot, \tau, u_{\tau}\right) u_{\tau} \text { weakly in } L^{2}(\tau, T ; W), \forall t \geq \tau .
\end{aligned}
$$

Lemma 4.1 Assume $f \in L^{2}(R, H)$, then there exists a global uniform (w.r.t. $f \in \Sigma$ ) absorbing set $B_{0}$ of the process $\left\{U_{f}\left(t, \tau, u_{\tau}\right)\right\}$. 
Proof. By the Faedo-Galerkin method, the standard elliptic operator theory and the Poincaré inequality, we get that $u$ belongs to $L^{2}((\tau, T) ; D(A)) \cap L^{\infty}((\tau, T)$; W), then using the Gronwall inequality and similar energy method to the proof of Theorem 3.1 in Qin, Yang and Liu [10], we can deduce the boundedness of $u$ and the existence of absorbing set.

Lemma 4.2 Under the condition of $f \in L^{2}(R, H), u_{\tau} \in W$, the process $\left\{U_{f}\left(t, \tau, u_{\tau}\right)\right\}$ generated by the global solutions for problem (1.1)-(1.4) is asymptotically compact in W.

Proof. For any initial data $u_{\tau}^{i} \in B_{0}(i=1,2)$, let $u^{i}(t, x)$ be the corresponding solutions to the symbols $f^{i}$ with $u_{\tau}^{i}$, that is, $u^{i}(t)$ is the solution of the problem (3.8)-(3.11). Denote $A=-\Delta$ and $w(t)=u^{1}(t)-u^{2}(t)$, then $w(t)$ satisfies the equivalent abstract equations (3.13)-(3.14).

Setting

$$
E_{w}(t)=\frac{1}{2} \int_{\Omega}|\nabla w(t)|^{2} \mathrm{~d} x+\frac{\alpha^{2}}{2} \int_{\Omega}|A w(t)|^{2} \mathrm{~d} x .
$$

Multiplying (3.13) by $A w$ and integrating over $[s, T] \times \Omega$, we deduce

$$
\begin{aligned}
& E_{w}(T)-E_{w}(s)+v \int_{s}^{T} \int_{\Omega}|A w(h)|^{2} \mathrm{~d} x \mathrm{~d} h \\
& +\int_{s}^{T} \int_{\Omega}\left(B\left(u^{1}(h)\right)-B\left(u^{2}(h)\right)\right) A w(h) \mathrm{d} x \mathrm{~d} h \\
& =\int_{s}^{T} \int_{\Omega}\left(f^{1}(h)-f^{2}(h)\right) A w(h) \mathrm{d} x \mathrm{~d} h,
\end{aligned}
$$

where $\tau \leq s \leq T$. Then we have

$$
\begin{aligned}
v \int_{\tau}^{T} \int_{\Omega}|A w(h)| \mathrm{d} x \mathrm{~d} h \leq & E_{w}(\tau)-\int_{\tau}^{T} \int_{\Omega}\left(B\left(u^{1}(h)\right)-B\left(u^{2}(h)\right)\right) A w(h) \mathrm{d} x \mathrm{~d} h \\
& +\int_{\tau}^{T} \int_{\Omega}\left(f^{1}(h)-f^{2}(h)\right) A w(h) \mathrm{d} x \mathrm{~d} h .
\end{aligned}
$$

Hence,

$$
\begin{aligned}
\int_{\tau}^{T} E_{w}(s) \mathrm{d} s= & \int_{\tau}^{T}\left(\frac{1}{2} \int_{\Omega}|A w(s)|^{2} \mathrm{~d} x+\frac{\alpha^{2}}{2} \int_{\Omega}|A w(s)|^{2} \mathrm{~d} x\right) \mathrm{d} s \\
\leq C & \int_{\tau}^{T} \int_{\Omega}|A w(s)|^{2} \mathrm{~d} x \mathrm{~d} s \\
\leq C & E_{w}(\tau)-\int_{\tau}^{T} \int_{\Omega}\left(B\left(u^{1}(s)\right)-B\left(u^{2}(s)\right)\right) A w(s) \mathrm{d} x \mathrm{~d} s \\
& \left.+\int_{\tau}^{T} \int_{\Omega}\left(f^{1}(s)-f^{2}(s)\right) A w(s) \mathrm{d} x \mathrm{~d} s\right] .
\end{aligned}
$$


Integrating (4.4) over $[\tau, T]$ with respect to $s$, we get

$$
\begin{aligned}
T E_{w}(T) & +v \int_{\tau}^{T} \int_{s}^{T} \int_{\Omega}|A w(h)|^{2} \mathrm{~d} x \mathrm{~d} h \mathrm{~d} s \\
\leq & \int_{\tau}^{T} E_{w}(s) \mathrm{d} s-\int_{\tau}^{T} \int_{s}^{T} \int_{\Omega}\left(B\left(u^{1}(h)\right)-B\left(u^{2}(h)\right)\right) A w(h) \mathrm{d} x \mathrm{~d} h \mathrm{~d} s \\
& +\int_{\tau}^{T} \int_{s}^{T} \int_{\Omega}\left(f^{1}(h)-f^{2}(h)\right) A w(h) \mathrm{d} x \mathrm{~d} h \mathrm{~d} s \\
\leq & C\left[E_{w}(\tau)-\int_{\tau}^{T} \int_{\Omega}\left(B\left(u^{1}(s)\right)-B\left(u^{2}(s)\right)\right) A w(s) \mathrm{d} x \mathrm{~d} s\right. \\
& \left.+\int_{\tau}^{T} \int_{\Omega}\left(f^{1}(s)-f^{2}(s)\right) A w(s) \mathrm{d} x \mathrm{~d} s\right] \\
& -\int_{\tau}^{T} \int_{s}^{T} \int_{\Omega}\left(B\left(u^{1}(h)\right)-B\left(u^{2}(h)\right)\right) A w(h) \mathrm{d} x \mathrm{~d} h \mathrm{~d} s \\
& +\int_{\tau}^{T} \int_{s}^{T} \int_{\Omega}\left(f^{1}(h)-f^{2}(h)\right) A w(h) \mathrm{d} x \mathrm{~d} h \mathrm{~d} s .
\end{aligned}
$$

If we set

$$
\begin{aligned}
& C_{0}=C E_{w}(\tau), \\
& \qquad \begin{aligned}
\phi\left(u_{0}^{1}, u_{0}^{2} ; g^{1}(t), g^{2}(t)\right)=C[- & \int_{\tau}^{T} \int_{\Omega}\left(B\left(u^{1}(s)\right)-B\left(u^{2}(s)\right)\right) A w(s) \mathrm{d} x \mathrm{~d} s \\
& \left.+\int_{\tau}^{T} \int_{\Omega}\left(f^{1}(s)-f^{2}(s)\right) A w(s) \mathrm{d} x \mathrm{~d} s\right] \\
& -\int_{\tau}^{T} \int_{s}^{T} \int_{\Omega}\left(B\left(u^{1}(h)\right)-B\left(u^{2}(h)\right)\right) A w(h) \mathrm{d} x \mathrm{~d} h \mathrm{~d} s \\
& +\int_{\tau}^{T} \int_{s}^{T} \int_{\Omega}\left(f^{1}(h)-f^{2}(h)\right) A w(h) \mathrm{d} x \mathrm{~d} h \mathrm{~d} s,
\end{aligned}
\end{aligned}
$$

then we have

$$
E_{w}(T) \leq \frac{C_{0}}{T}+\frac{1}{T} \phi\left(u_{0}^{1}, u_{0}^{2} ; f^{1}(t), f^{2}(t)\right)
$$

Since the family of the processes has a uniformly bounded absorbing set, we choose $T$ large enough such that

$$
\frac{C_{0}}{T} \leq \varepsilon,
$$

i.e., $T \geq \frac{C_{0}}{\varepsilon}$.

Let $u^{n}, u^{m}$ be the solutions with respect to the initial data $u_{0}^{n}, u_{0}^{m}$ and symbols $f^{n}(t)$, $f^{m}(t) \in \Sigma, m, n=1,2, \ldots$ respectively. Then we can obtain

$$
\begin{aligned}
& \lim _{n \rightarrow \infty} \lim _{m \rightarrow \infty} \int_{\tau}^{T} \int_{s}^{T} \int_{\Omega}\left(f^{n}(h)-f^{m}(h)\right)\left(A u^{n}(s)-A u^{m}(s)\right) \mathrm{d} x \mathrm{~d} h \mathrm{~d} s=0, \\
& \lim _{n \rightarrow \infty} \lim _{m \rightarrow \infty} \int_{\tau}^{T} \int_{\Omega}\left(f^{n}(s)-f^{n}(s)\right)\left(A u^{n}(s)-A u^{m}(s)\right) \mathrm{d} x \mathrm{~d} s=0
\end{aligned}
$$


and

$$
\begin{aligned}
& \lim _{n \rightarrow \infty} \lim _{m \rightarrow \infty} \int_{\tau}^{T} \int_{\Omega}\left(B\left(u^{n}(s)\right)-B\left(u^{m}(s)\right)\right)\left(A u^{n}(s)-A u^{m}(s)\right) \mathrm{d} x \mathrm{~d} s \\
& =\lim _{n \rightarrow \infty} \lim _{m \rightarrow \infty} \int_{s}^{T} \int_{\Omega}\left(\left(u^{n}(s) \cdot \nabla\right) u^{n}(s)-\left(u^{m}(s) \cdot \nabla\right) u^{m}(s)\right) \\
& \times\left(A u^{n}(s)-A u^{m}(s)\right) \mathrm{d} x \mathrm{~d} s \\
& =\lim _{n \rightarrow \infty} \lim _{m \rightarrow \infty} \int_{s}^{T} \int_{\Omega}\left(\left(\left(u^{n}(s)-u^{m}(s)\right) \cdot \nabla\right) u^{n}(s)-\left(u^{m}(s) \cdot \nabla\right)\right. \\
& \left.\times\left(u^{m}(s)-u^{n}(s)\right)\right) \times\left(A u^{n}(s)-A u^{m}(s)\right) \mathrm{d} x \mathrm{~d} s \\
& =0, \\
& \lim _{n \rightarrow \infty} \lim _{m \rightarrow \infty} \int_{\tau}^{T} \int_{s}^{T} \int_{\Omega}\left(B\left(u^{n}(h)\right)-B\left(u^{m}(h)\right)\right)\left(A u^{n}(s)-A u^{m}(s)\right) \mathrm{d} x \mathrm{~d} h \mathrm{~d} s \\
& =\lim _{n \rightarrow \infty} \lim _{m \rightarrow \infty} \int_{\tau}^{T} \int_{s}^{T} \int_{\Omega}\left(\left(u^{n}(h) \cdot \nabla\right) u^{n}(h)-\left(u^{m}(h) \cdot \nabla\right) u^{m}(h)\right) \\
& \times\left(A u^{n}(s)-A u^{m}(s)\right) \mathrm{d} x \mathrm{~d} h \mathrm{~d} s \\
& =\lim _{n \rightarrow \infty} \lim _{m \rightarrow \infty} \int_{\tau}^{T} \int_{s}^{T} \int_{\Omega}\left(\left(\left(u^{n}(h)-u^{m}(h)\right) \cdot \nabla\right) u^{n}(h)-\left(u^{m}(h) \cdot \nabla\right)\right. \\
& \left.\times\left(u^{m}(h)-u^{n}(h)\right)\right) \times\left(A u^{n}(s)-A u^{m}(s)\right) \mathrm{d} x \mathrm{~d} h \mathrm{~d} s \\
& =0 .
\end{aligned}
$$

Hence $\phi\left(u_{0}^{1}, u_{0}^{2} ; f^{1}(t), f^{2}(t)\right) \in \operatorname{Contr}\left(B_{0}, \Sigma\right)$ for the above $T$. By Lemma 2.2 and the property of the functional $\langle\cdot, A \cdot\rangle+\alpha^{2}\langle A \cdot, A \cdot\rangle$, the conclusion holds.

Proof of Theorem 2.4 From Lemmas 4.1-4.2, we can deduce the result easily.

\section{Acknowledgements}

The work in part was supported by the NNSF of China (No. 11031003 and 10871040).

\section{Author details}

${ }^{1}$ College of Information Sciences and Technology, Donghua University, Songjiang, Shanghai, 201620, People's Republic of China ${ }^{2}$ College of Computer and Information Sciences, Anhui Polytechnique University, Wuhu, Anhui, 241000 People's Republic of China ${ }^{3}$ College of Mathematics and Information Science, Henan Normal University, Xinxiang, 453007, People's Republic of China ${ }^{4}$ Department of Applied Mathematics, Donghua University, Songjiang, Shanghai, 201620, People's Republic of China

\section{Authors' contributions}

The authors declare that the work was realized in collaboration with same responsibility. All authors read and approved the final manuscript.

\section{Competing interests}

The authors declare that they have no competing interests.

Received: 7 July 2011 Accepted: 28 November 2011 Published: 28 November 2011

\section{References}

1. Leray, J: Etude de diverses equations integrales nonlineaires et de quelques problemes que pose l'hydrodynamique. J Math Pures Appl. 12, 1-82 (1933)

2. Leray, J: Essai sur les mouvements plans d'un liquide visqueux que limitent des parois. J Math Pures Appl. 13, 331-418 (1934)

3. Leray, J: Essai sur le mouvement d'un liquide visqueux emplissant l'espace. Acta Math. 63, 193-248 (1934). doi:10.1007/ BF02547354

4. Hopf, E: Ueber die Anfangswertaufgable fur die hydrodynamischen Grundgleichungen. Math Nachr. 4, $213-231$ (1951)

5. Sell, GR: Global attractors for the three-dimensional Navier-Stokes equations. J Dyn Differ Equ. 8, 1-33 (1996). doi:10.1007/BF02218613

6. Chepyzhov, W, Vishik, MI: Evolution equations and their trajectory attractors. J Math Pures Appl. 76, 664-913 (1997) 
7. Kapustyan, OV, Valero, J: Weak and strong attractors for the 3D Navier-Stokes system. J Differ Equ. 240, 249-278 (2007). doi:10.1016/j.jde.2007.06.008

8. Kapustyan, OV, Kasyanov, PO, Valero, J: Pullback attractors for a class of extremal solutions of the 3D Navier-Stokes system. J Math Anal Appl. 373, 535-547 (2011). doi:10.1016/j.jmaa.2010.07.040

9. Kalantarov, VK, Titi, ES: Global attractors and determining models for the 3D Navier-Stokes-Voight equations. Chin Ann Math. 30(B), 697-714 (2009)

10. Qin, Y, Yang, X, Xin, Liu: Uniform attractors for a 3D non-autonomous Navier-Stokes-Voight Equations, Preprint. (2010, in press)

11. Yue, G, Zhong, C: Attractors for autonomous and nonautonomous 3D Navier-Stokes-Voight equations. Discret Contin Dyn Syst Ser B. 2011(6-3), 985-1002 (2011)

12. Babin, AV, Vishik, Ml: Attractors of Evolutionary Equations, Studies in Mathematics and Its Applications. North-Holland, Amesterdam, London, New York, Tokyo25 (1992)

13. Chepyzhov, W, Vishik, Ml: Attractors for Equations of Mathematical Physics. Providence, Rl: American Mathematical Society (2001)

14. Chueshov, I: Introduction to the Theory of Infinite-Dimensional Dissipative Systems, ACTA. (2002)

15. Feifeisl, E: Dynamics of Viscous Compressible Fluids. Oxford University Press, Oxford (2004)

16. Hale, JK: Asymptotic Behavior of Dissipative Systems. American Mathematical Society, Providence, RI (1988)

17. Hou, $Y, L i, K$ : The uniform attractors for the $2 \mathrm{D}$ non-autonomous Navier-Stokes flow in some unbounded domain Nonlinear Anal TMA.58, 609-630

18. Ladyzhenskaya, OA: The mathematical Theory of Viscous Incompressible Flow. Gordon and Breach, New York (1969)

19. Ladyzhenskaya, OA: Attractors for Semigroup and Evolution Equations. Cambridge University Press, Cambridge (1991)

20. Lu, SS, Wu, H, Zhong, CK: Attractors for non-autonomous 2D Navier-Stokes equations with normal external forces. Disc Cont Dyn Syst. 13(3), 701-719 (2005)

21. Miranville, A, Wang, X: Attractors for Non-autonomous nonhomogenerous Navier-Stokes equations. pp. 1047-01061. Nonlinearity10(5), (1997)

22. Qin, Y: Nonlinear Parabolic-Hyperbolic Coupled Systems and Their Attractors. Operator Theory, Advances and Applications. Birkhäuser, Basel-Boston-Berlin184

23. Qin, Y: Universal attractor in $H^{4}$ for the nonlinear one-dimensional compressible Navier-Stokes equations. J Differ Equ. 207(1), 21-72 (2004). doi:10.1016/j.jde.2004.08.022

24. Robinson, JC: Infinite-Dimensional Dynamics Systems. Cambridge University Press, Cambridge (2001)

25. Sell, GR, You, Y: Dynamics of Evolutionary Equations. Springer, New York (2002)

26. Temam, R: Infinite-Dimensional Dynamical Systems in Mechanics and Physics. Springer, Berlin (1997)

27. Temam, R: Navier-Stokes Equations, Theory and Numerical Analysis. North Holland, Amsterdam (1979)

28. Yang, L: Uniform attractor for non-autonomous hyperbolic equation with critical exponent. Appl Math Comput. 203, 895-902 (2008). doi:10.1016/j.amc.2008.05.113

doi:10.1186/1687-2770-2011-49

Cite this article as: Dou et al:: Remarks on uniform attractors for the 3D non-autonomous Navier-Stokes-Voight equations. Boundary Value Problems 2011 2011:49.

\section{Submit your manuscript to a SpringerOpen ${ }^{\ominus}$ journal and benefit from:}

Convenient online submission

- Rigorous peer review

- Immediate publication on acceptance

- Open access: articles freely available online

- High visibility within the field

- Retaining the copyright to your article

Submit your next manuscript at $\gg$ springeropen.com 